\title{
Genetic variation of RAPD markers in a Picea abies Karst. population
}

\author{
GABRIELE BUCCI* \& PAOLO MENOZZI \\ Istituto di Ecologia, Università di Parma, Viale delle Seienze, 43100 Parma, Italy
}

\begin{abstract}
We report the results of the screening of 20 mapped RAPD loci over haploid megagametophytes from 48 individual trees of an Italian population of Norway spruce. LOD scores for 190 pairs of loci were calculated over families of megagametophytes from 'informative' trees, and linkage relationships between pairs of markers were analysed. Single-locus genotypes were inferred from haploid progeny-array data and were used to estimate linkage disequilibrium between loci. Significant deviations from equilibrium did not appear to be randomly distributed over loci. Most of the markers analysed showed an excess of heterozygotes and five out of 20 RAPD markers showed significant deviations from Hardy-Weinberg equilibrium. A weak spatial structure was detected by spatial autocorrelation at the scale considered $(0-180 \mathrm{~m})$, suggesting a lack of 'family structure' arising from isolation by distance. Nonrandom distribution of the genetic variability was limited to some loci. Di- and tri-locus spatial autocorrelation of loci showing linkage disequilibrium was also carried out.
\end{abstract}

Keywords: genetic structure, linkage, linkage disequilibrium, Norway spruce, RAPDs, spatial autocorrelation.

\section{Introduction}

Microevolutionary processes driving the amount and the organization of genetic variability and genetic structure within populations of forest trees have been extensively investigated in the last two decades thanks to allozymic markers (for a review see Müller-Starck et al., 1992). The genetic information obtained by the limited number of suitable allozymic loci has been considered inadequate for several purposes (Leigh Brown, 1989; Wagner, 1992), given the restricted, nonrandom genome sampling (Bergmann, 1991) and the low sensitivity to mutation at the DNA level (Kreitmann, 1983). Moreover, linkage relationships between allozymic markers are not always well-known for several tree species, leading to (eventually) biased estimation of the genetic parameters of the population.

The RAPD technique has been indicated as one of the most powerful methods for obtaining a large number of genetic markers in a wide range of species (Williams et al., 1990; Hadrys et al., 1992). The genetic origin of the RAPD bands is usually verified in the progeny from controlled crosses (Reiter et al., 1992). In conifers, the Mendelian inheritance of the RAPD bands may be verified by analysing a suitable number of (haploid) megagametophytes from a single tree

*Correspondence.
(Neale \& Sederoff, 1991; Tulsieram et al., 1992; Nelson et al., 1993). The high number of random primers of arbitrary sequence available may allow an extensive, random sampling of the genome (Paran et al., 1991), while the outstanding degree of variability detected at the DNA level may increase the amount of genetic information.

On the other hand, RAPDs have been reported to be sensitive to amplification conditions (Ellsworth et al., 1993) and to different genomic backgrounds, showing nonparental bands (Ayliffe et al., 1994) and/ or ambiguous linkage relationships (W. L. Nance, personal communication). The availability of genetic linkage maps from different individuals of the same species would allow the comparison of the chromosomal localization of the markers (genomic synteny). Confirmation of the reliability and usefulness of RAPDs in investigations of wild populations needs to be addressed for their proper utilization as conventional genetic markers.

Once confirmed, the availability of a large number of markers obtained by RAPDs could usefully be exploited in the study of the organization of genetic variability of forest tree populations. Improved estimates of genetic correlation between individuals could greatly enhance the detection of genetic structure within populations (Wagner, 1992). Detailed genetic profiles obtained by monitoring large genomic regions 
may allow multilocus genotypes to be better tracked along different environmental gradients (Allard et al., 1972; Hamrick \& Allard, 1972; Mitton et al., 1980). Association between spatial patterns of genetic variation and spatial patterns of environmental heterogeneity (Epperson, 1990), as revealed by spatial autocorrelation techniques (Cliff \& Ord, 1973; Sokal \& Oden, 1978), could lead to the identification of interacting sets of loci or peculiar allelic configurations involved in the underlying microevolutionary processes. On the other hand, lowered recombination rates between markers because of tight linkage could eventually result, in particular circumstances, in a multilocus spatial association of genotypes (Slatkin \& Arter, 1991) arising from a 'hitch-hiking effect' (Hastings, 1990).

In this study, we report results of the screening of previously mapped RAPD loci (Binelli \& Bucci, 1994) over haploid megagametophytes from 48 individual trees of Picea abies Karst. Such progeny-array data were used to investigate the linkage relationships of the markers over the whole population by the LOD score method (Morton, 1955). Single-locus genotypes inferred from haploid data were then analysed by means of spatial autocorrelation techniques, in order to investigate the spatial genetic structure within a natural population of Norway spruce.

\section{Materials and methods}

\section{DNA extraction and amplification}

Forty-eight adult-stage individuals of Norway spruce (Picea abies) from the Campolino stand (Northern Apennines, Italy) were sampled at random. Cones were harvested and left some days at room temperature until the scales were open. Seeds were then collected separately and stored at $4^{\circ} \mathrm{C}$.

Six megagametophytes per tree were isolated from germinated seeds using scalpel and forceps under a low-magnification stereomicroscope, and stored in a microcentrifuge tube at $-80^{\circ} \mathrm{C}$ until DNA extraction, performed following the CTAB method (Wagner et al., 1987), adjusted in volume for small tissue samples. DNA concentration for all samples was determined by fluorometric assays (Cesarone et al., 1979).

DNA amplification was carried out using Thermowell C 96-wells polycarbonate V-bottom shaped microtitre plates (Costar) in a MJ Research PTC-100/ 96 thermal cycler. Amplification conditions and temperature profiles were chosen according to conditions previously reported (Binelli \& Bucci, 1994). Primers (10-mer) of arbitrary sequence were used. The amplified products were separated by 2 per cent agarose gel electrophoresis in TBE 1X, stained with ethidium bromide and photographed using a Polaroid camera. Bands were scored by two persons independently.

\section{Choice of the markers}

Four 10-mer primers producing 20 RAPD markers in the linkage map previously reported (Binelli \& Bucci, 1994 ) were used. Of these 20 markers, pairs $25 / 26,27 /$ 28 and $24 / 178$ have been found to be linked by the above authors $(\mathrm{rf}=0.031,0.016$ and 0.189 , respectively), the others being evenly spaced within and between linkage groups. The marker bands chosen for the screening of the genetic variability over the whole population are shown in Table 1.

The overall number of amplification reactions performed in this study was 1152 (6 megagametophytes per tree $\times 48$ trees $\times 4$ primers used). Reproducibility of the previously mapped markers was verified for every amplification round by including DNA of 6 megagametophytes from the tree used to build a RAPD linkage map (Binelli \& Bucci, 1994) and comparing the amplification patterns by molecular weight. Single-locus genotypes of the sampled trees were inferred from haploid progeny-array data.

\section{Segregation analysis and estimation of the population parameters}

For a heterozygous tree, the number $x$ of megagametophytes showing a given marker band follows a binomial distribution:

$$
\frac{n !}{x ! \cdot(n-x) !} p^{n}(1-p)^{n-x}
$$

where $p$ is the likelihood of the alternative events $(0.5$, assuming no segregation distortion) and $n$ is the total number of megagametophytes within the family. For a given locus, a tree was considered heterozygous when $1 \leq x \leq 5$ out of 6 megagametophytes. The binomial could be fitted only for heterozygotes and classes $x=0$ and $x=6$ were then excluded from the test. Goodnessof-fit was verified for each locus by pooling data from all the heterozygous trees. Given the small number of observations for some of the loci analysed we used the Kolmogorov-Smirnov test $(\alpha=0.05)$. The fit to the binomial distribution was also tested by pooling data over all the heterozygous trees and over loci and using a chi-square test $(\alpha=0.05)$.

The unbiased gene diversity over the whole population was estimated following Nei (1987):

$H_{\exp }=\frac{2 n}{2 n-1}\left(1-\Sigma p_{i}^{2}\right)$. 
Table 1 Analysis of RAPD markers in a population of Norway spruce

\begin{tabular}{|c|c|c|c|c|c|c|c|}
\hline Label & Marker & $N$ & $p$ & $\chi^{2}$ & $H_{\exp }$ & $H_{\text {obs }}$ & $F_{\text {IS }}$ \\
\hline 23 & C184-0240 & 39 & 0.192 & 0.035 & 0.315 & 0.282 & 0.092 \\
\hline 24 & C184-0250 & 32 & 0.063 & 0.005 & 0.119 & 0.125 & -0.067 \\
\hline 25 & C184-0340 & 43 & 0.151 & 0.111 & 0.260 & 0.302 & -0.178 \\
\hline 26 & C184-0350 & 43 & 0.221 & 0.118 & 0.348 & 0.395 & -0.148 \\
\hline 27 & C184-0490 & 43 & 0.221 & 0.079 & 0.348 & 0.302 & 0.122 \\
\hline 28 & C184-0500 & 41 & 0.829 & 0.162 & 0.287 & 0.341 & -0.206 \\
\hline 29 & C184-0680 & 42 & 0.679 & 0.524 & 0.441 & 0.548 & -0.255 \\
\hline 30 & C184-1200 & 39 & 0.910 & 0.018 & 0.166 & 0.179 & -0.099 \\
\hline 45 & C266-0820 & 38 & 0.408 & $8.429^{* *}$ & - & - & - \\
\hline 46 & C266-1205 & 37 & 0.230 & 0.427 & 0.359 & 0.459 & -0.298 \\
\hline 47 & $\mathrm{C} 266-1350$ & 35 & 0.300 & $6.428^{*}$ & - & - & - \\
\hline 48 & C266-1810 & 35 & 0.400 & 3.353 & 0.487 & 0.629 & -0.310 \\
\hline 49 & C266-2000 & 37 & 0.824 & 0.091 & 0.294 & 0.243 & 0.160 \\
\hline 111 & C631-0395 & 34 & 0.441 & $10.320^{* *}$ & - & - & - \\
\hline 112 & C631-0746 & 32 & 0.938 & 0.005 & 0.119 & 0.125 & -0.067 \\
\hline 113 & C631-1078 & 33 & 0.621 & 0.385 & 0.478 & 0.576 & -0.223 \\
\hline 114 & С631-2061 & 30 & 0.500 & 0.133 & 0.508 & 0.533 & -0.067 \\
\hline 178 & OX20-0510 & 40 & 0.613 & 1.786 & 0.481 & 0.575 & -0.211 \\
\hline 179 & OX20-0550 & 37 & 0.500 & $22.730^{* * *}$ & - & - & - \\
\hline 180 & OX20-0650 & 35 & 0.514 & $17.930^{* * *}$ & - & - & - \\
\hline \multirow[t]{3}{*}{20} & Overall & 745 & & & & & \\
\hline & Average & 37.25 & & & 0.334 & 0.381 & -0.136 \\
\hline & $\mathrm{SE}$ & 0.89 & & & 0.034 & 0.044 & - \\
\hline
\end{tabular}

Labels refer to the Norway spruce linkage map reported elsewhere (Binelli \& Bucci, 1994; see also Bucci et al., 1995). Markers are identified by the name of the relative primer $(\mathrm{C}=$ University of British Columbia, $\mathrm{BC}, \mathrm{Canada} ; \mathrm{O}=\mathrm{Operon}$ Technologies, Alameda, CA) and their approximate molecular weight.

Number of individuals $(N)$, frequencies of the ' 1 ' allele $(p)$, and $\chi^{2}$ for deviations from the Hardy-Weinberg equilibrium (HWE) are given for the 20 RAPD markers analysed in this investigation.

$* P<0.05,{ }^{* *} P<0.01,{ }^{* * *} P<0.001$.

Expected $\left(H_{\text {exp }}\right)$ and observed $\left(H_{\text {obs }}\right)$ heterozygosity, fixation index $\left(F_{\text {IS }}\right)$, averages and standard errors $(\mathrm{SE})$ were calculated for the 15 markers in agreement with Hardy-Weinberg equilibrium.

A maximum likelihood estimate of inbreeding within the population was calculated following $\mathrm{Nei}(1987)$ :

$F_{\mathrm{IS}}=\frac{4 n_{11} n_{00}-n_{10}^{2}}{\left(2 n_{11}+n_{10}\right)\left(2 n_{00}+n_{10}\right)}$

where $n_{11}, n_{00}$ and $n_{10}$ are the number of homozygous trees for the allele ' $I$ ', the number of the homozygous trees for the allele ' $O$ ' and the number of heterozygous trees, respectively. The average inbreeding coefficient over all loci and individuals was estimated as the weighted average of the single-locus $F_{\text {Is }}$ estimates (Nei, 1987).

\section{Linkage analysis over the whole population}

Linkage analysis between RAPD markers over the whole population was restricted to pairs of informative genotypes, i.e. mother trees shown to be doubly heterozygous for a given pair of loci (Mather, 1951). Analysis was also restricted to heterozygous trees showing at least 5 dilocus unambiguous, haploid scores $(n \geq 5)$.

Estimation of the linkage between markers over the whole population was carried out considering each family of megagametophytes as the offspring of a phase-unknown double backcross mating between a double heterozygote mother (' $10 / 10$ ' or ' $10 / 01$ ', being the markers in coupling- or in repulsion-phase, respectively) and a fictitious double homozygous father for the recessive alleles ('00/00'). The observed LOD scores for each pair of RAPD loci were then estimated at the selected recombination fraction by means of the following formula (Ott, 1991):

$$
\begin{aligned}
Z\left(\theta_{i}\right)= & (m-1) \log _{10}(2)+\log _{10}\left[\theta_{i}^{k}\left(1-\theta_{i}\right)^{m-k}\right. \\
& \left.+\theta_{i}^{m-k}\left(1-\theta_{i}\right)^{k}\right],
\end{aligned}
$$

c The Genetical Society of Great Britain, Heredity, 75, 188-197. 
where $\theta_{i}$ is a fixed recombination value $\left(\theta_{i}=0.001\right.$, $0.01,0.05,0.1,0.15,0.2,0.25,0.3,0.35,0.4,0.45$ ) between the $i$ th pair of loci, $m$ is the number of offspring (megagametophytes) per family and $k$ is the number of crossovers observed. LOD scores for each pair of loci at the corresponding $\theta_{i}$ were then added over families of megagametophytes. Linkage between pairs of markers was considered significant when $Z\left(\theta_{i}\right) \geq 3.0\left(\alpha \leq 10^{-3}=0.001\right)$. Homogeneity of the recombination fraction between families of megagametophytes was verified by the $M$-test (Morton, 1955). Independence of loci was also verified by calculating the following statistic (Gerber \& Rodolphe, 1994):

$S=\sum_{i=1}^{m} \frac{2 x_{i}-n_{i}}{n_{i}}$,

where $m$ is the number of informative trees for both loci. Under the null hypothesis of independence of loci $\left(\theta_{i}=0.5\right)$, the $S$ statistic has a chi-square distribution with $m$ degrees of freedom (Gerber \& Rodolphe, 1994).

\section{Linkage disequilibrium}

Analysis of the dilocus disequilibrium between RAPD markers was carried out using single-locus genotype data from all the trees (homo- and heterozygous) showing at least five unambiguous haploid scores $(n \geq 5)$.

Deviation of the frequencies of the dilocus gametic genotypes from the product of the single allele frequencies was estimated using the method of Cockerham \& Weir (1977):

$D=\frac{4 n_{1}+2 n_{2}+2 n_{3}+n_{4}}{2 n}-2 p_{1} p_{2}$,

where $n_{1}, n_{2}, n_{3}$, and $n_{4}$ are the number of diploid genotypes showing the dilocus genotype ' $11 / 11$ ', '11/ 10 ', ' $10 / 11$ ', ' $10 / 10$ ', respectively, $n$ is the total number of individuals analysed and $p_{1}, p_{2}$ are the frequencies of the allele ' 1 ' at the two loci considered. Variances of the estimates were calculated using the following formula (Cockerham \& Weir, 1977):

$$
\begin{aligned}
V(D)= & {\left[p_{1}\left(1-p_{1}\right) p_{2}\left(1-p_{2}\right)\right.} \\
& \left.+0.5\left(1-2 p_{1}\right)\left(1-2 p_{2}\right) D+\left(D^{2} / n\right)\right] n .
\end{aligned}
$$

Differences from equilibrium were verified by the chisquare test (Weir, 1990) for each pair of loci. The power of the test (i.e. the likelihood $\beta$ of obtaining again a significant value of $D$ on drawing another random sample from the population studied) was calculated following Weir (1990).

\section{Spatial distribution of the genetic variability detected}

The analysis of the spatial distribution of the genetic variability was carried out by spatial autocorrelation (Sokal \& Oden, 1978). The total number of joins was $1128[=(48 \cdot 47) / 2]$. Five distance classes with even number of joins between individuals in each class were defined (for distance class $I=0-28.7 \mathrm{~m}$; for $I I=28.8-51.2 \mathrm{~m} ; \quad$ for $\quad I I I=51.3-71.9 \mathrm{~m}$; for $I V=72-101.1 \mathrm{~m}$; for $V=101.2-174.4 \mathrm{~m})$. Standard Normal Deviates (s.n.d.) of the frequencies of the observed genotypic pairs from the expectation based on the genotypic frequencies were calculated for each of the five distance classes. Dilocus and trilocus spatial autocorrelation was carried out considering (recoding) the genotype made up by combination of alleles at two or three loci.

\section{Results}

\section{Segregation analysis and estimation of the population parameters}

Out of 960 possible single-locus genotypes inferred from the analysis of six megagametophytes (20 loci in 48 mother trees), 745 ( 77.6 per cent) were identified with a probability of 94 per cent (at least five unambiguous scores) and used for further analysis. The remaining 215 single-locus genotypes were discarded because of faintly stained bands in two or more megagametophytes from the same tree. An average of $37.25 \pm 0.86$ individuals per locus were identified, with $15.52 \pm 0.73$ single-locus genotypes per individual.

Of 349 heterozygous single-locus genotypes detected, 225 ( 64.5 per cent) were identified by six unambiguous scores and 124 were based on five scores out of six megagametophytes. The distribution of the 225 unambiguous segregation ratios within families (Fig. 1) showed no significant difference from the expected binomial distribution $\left(\chi_{4}^{2}=3.748 ; P>0.05\right)$. For each marker, segregation ratios within families fit the expected binomial distribution for Mendelian traits (Kolmogorov-Smirnov $D_{\max }, \quad P>0.05-$ data not shown).

Five out of the 20 RAPD markers showed significant deviations from Hardy-Weinberg equilibrium (d.f. $=1 ; P<0.05-$ Table 1), owing to an excess of heterozygotes (Fig. 2). These RAPD markers were not used in further analysis.

Expected genetic diversity ranged from 0.119 to 0.508 with a mean of $0.334 \pm 0.034$. For all but three cases $(23,27$ and 49) single-locus inbreeding estimates $\left(F_{\text {IS }}\right)$ were slightly negative, while the average $F_{\text {IS }}$ over the whole population was -0.136 , indicating an excess of heterozygotes (Table 1). 


\section{Linkage relationships over the whole population}

A total of 1970 informative haploid scores (i.e. marker scores from informative loci) out of 2094 (six haploid megagametophytes $\times 349$ heterozygous single-locus genotypes unambiguously scored for at least five megagametophytes) were analysed. On average, the number of marker scores analysed per pair of loci was $42.89 \pm 0.13$, while the mean number of families of megagametophytes per pair of loci was $7.82 \pm 0.02$.

The results of the linkage analysis between pairs of RAPD markers are set out in Table 2. Analysis of the LOD scores confirmed markers 27 and 28 to be significantly linked $\left[Z\left(\theta_{0.1}\right)=3.523 ; P=0.00029 ; M\right.$-test $\left.\chi_{6}^{2}=4.997\right]$. Not surprisingly, these markers had been found to be tightly linked ( $\mathrm{rf}=0.016$, linkage group $\mathrm{B}$ ) in the genetic map previously reported (Binelli \& Bucci, 1994). On the other hand, in this study markers $25 / 26\left[Z\left(\theta_{0.3}\right)=1.23 ; P=0.058\right]$ and markers $24 / 178$

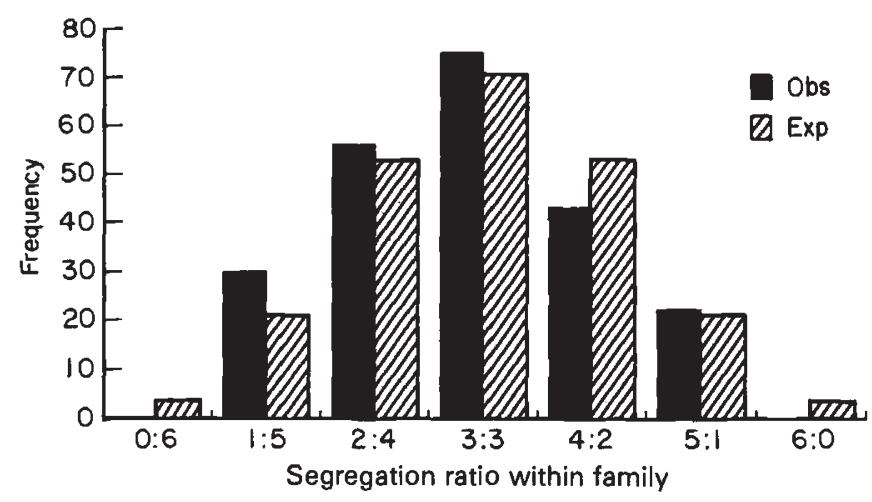

Fig. 1 Frequency of segregation ratios observed within families (see text) and binomial expectations. No significant differences were found between the above distributions $\left(N=225 ; \chi_{4}^{2}=3.784 ; P>0.05\right)$.
$\left[Z\left(\theta_{0.3}\right)=0.301 ; P=0.50\right]$ did not show significant linkage, although independence of loci $25 / 26$ over the population was rejected $\left(S=\chi_{11}^{2}=24.268\right.$; $0.01 \leq P \leq 0.02$ ). The above pairs of loci had been found to be linked in the previous genetic map of Picea abies ( $\mathrm{r} f=0.031$, linkage group $\mathrm{R}$ and $\mathrm{rf}=0.189$, linkage group $\mathrm{K}$, respectively). As expected for unlinked markers, all the other dilocus combinations provide no evidence for linkage.

Because of the low number of megagametophytes per family - that does not allow the coupling- vs. repulsion-double heterozygotes to be unmistakably distinguished - we estimated gametic disequilibrium between loci from genotypic frequencies inferred from progeny-array data. The results obtained from the dilocus genotype analysis are shown in Table 2.

Deviation of the observed gametic frequencies from the product of the single allele frequencies turned out to be significant for five ( 2.63 per cent) out of 190 possible pairs of loci, a proportion smaller than expected by chance alone. Nevertheless, significant deviations from equilibrium do not appear to be randomly distributed over loci, mainly involving the loci 27, 28 and 178 (Table 2). One of the significant $D$ values involved a pair of linked markers (i.e. markers 27/28: $\quad D=-0.07167 \pm 0.00004, \quad \chi_{1}^{2}=12.451$, $P<0.001)$ with a power of the chi-square test larger than 0.95 . All the other significant $D$ values involved pairs of unlinked loci and showed a power of the test lower than 0.90 .

\section{Spatial distribution of the genetic variability}

By means of single-locus spatial autocorrelation techniques, we only detected 34 (9.57 per cent) significant

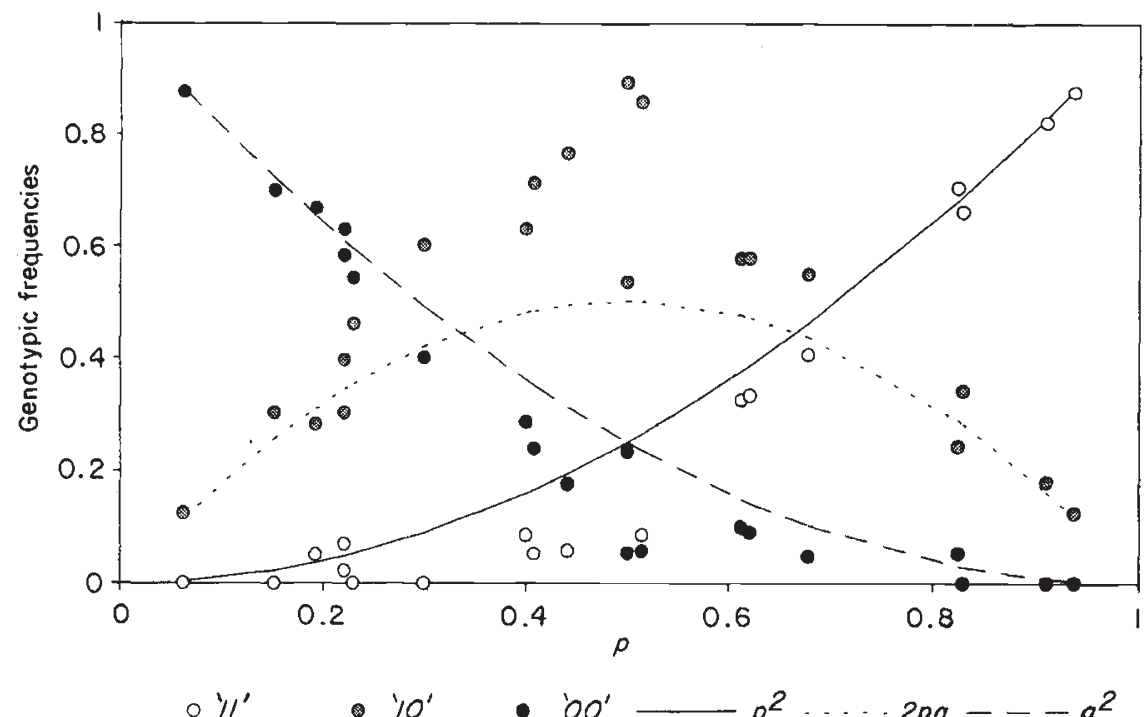

Fig. 2 Observed vs. expected genotypic frequencies for the 20 RAPD loci utilized in this investigation as a function of the frequency of the ' 1 ' allele. Most of the observed deviations from Hardy-Weinberg equilibrium result from an excess of heterozygotes. 
Table 2 Linkage relationships between the RAPD markers over the whole population of Norway spruce

\begin{tabular}{|c|c|c|c|c|c|c|c|c|c|c|c|c|c|c|c|c|c|c|c|c|}
\hline & 23 & 24 & 25 & 26 & 27 & 28 & 29 & 30 & 45 & 16 & 17 & 48 & 19 & 111 & 112 & 113 & 114 & 178 & 179 & 180 \\
\hline 23 & • & $\begin{array}{r}0.026 \\
(0.35)\end{array}$ & $\begin{array}{l}0.352 \\
(0.3)\end{array}$ & $\begin{array}{l}0.441 \\
(0.3)\end{array}$ & $\begin{array}{r}-0.008 \\
(0.45)\end{array}$ & $\begin{array}{c}0.249 \\
(0.25)\end{array}$ & $\begin{array}{l}-0.047 \\
(0.45)\end{array}$ & $\begin{array}{r}0.003 \\
(0.45)\end{array}$ & $\begin{array}{l}0.024 \\
(0.4)\end{array}$ & $\begin{array}{l}0.237 \\
(0.3)\end{array}$ & $\begin{array}{l}-0.035 \\
(0.45)\end{array}$ & $\begin{array}{l}-0.001 \\
(0.45)\end{array}$ & $\begin{array}{c}0.128 \\
(0.35)\end{array}$ & $\begin{array}{l}-0.005 \\
(0.45)\end{array}$ & $\begin{array}{l}0.103 \\
(0.3)\end{array}$ & $\begin{array}{l}-0.049 \\
(0.45)\end{array}$ & $\begin{array}{r}0.003 \\
(0.45)\end{array}$ & $\begin{array}{l}0.174 \\
(0.3)\end{array}$ & $\begin{array}{r}-0.01 \\
(0.45)\end{array}$ & $\begin{array}{r}0.159 \\
(0.35)\end{array}$ \\
\hline 24 & $0.362^{n}$ & - & $\begin{array}{l}-0.004 \\
(0.45)\end{array}$ & $\begin{array}{l}0.135 \\
(0.3)\end{array}$ & $\begin{array}{l}-0.018 \\
(0.45)\end{array}$ & $\begin{array}{l}-0.035 \\
(0.45)\end{array}$ & $\begin{array}{r}0.512 \\
(0.25)\end{array}$ & $\begin{array}{l}-0.013 \\
(0.45)\end{array}$ & $\begin{array}{r}-0.01 \\
(0.45)\end{array}$ & $\begin{array}{r}-0.01 \\
(0.45)\end{array}$ & $\begin{array}{l}-0.022 \\
(0.45)\end{array}$ & $\begin{array}{l}-0.005 \\
(0.45)\end{array}$ & $\begin{array}{l}-0.022 \\
(0.45)\end{array}$ & $\begin{array}{l}-0.031 \\
(0.45)\end{array}$ & $\begin{array}{l}0.071 \\
(0.3)\end{array}$ & $\begin{array}{l}-0.004 \\
(0.45)\end{array}$ & $\begin{array}{l}-0.027 \\
(0.45)\end{array}$ & $\begin{array}{l}0.301 \\
(\mathbf{0 . 3 )}\end{array}$ & $\begin{array}{l}-0.018 \\
(0.45)\end{array}$ & $\begin{array}{r}0.042 \\
(0.35)\end{array}$ \\
\hline 25 & 0.314 & 0.082 & - & $\begin{array}{r}1.23^{n} \\
(0.25)\end{array}$ & $\begin{array}{c}0.026 \\
(0.35)\end{array}$ & $\begin{array}{l}-0.013 \\
(0.45)\end{array}$ & $\begin{array}{l}-0.045 \\
(0.45)\end{array}$ & $\begin{array}{l}-0.001 \\
(0.45)\end{array}$ & $\begin{array}{l}0.288 \\
(0.3)\end{array}$ & $\begin{array}{l}-0.021 \\
(0.45)\end{array}$ & $\begin{array}{l}-0.022 \\
(0.45)\end{array}$ & $\begin{array}{l}-0.018 \\
(0.45)\end{array}$ & $\begin{array}{l}-0.009 \\
(0.45)\end{array}$ & $\begin{array}{l}0.029 \\
(0.4)\end{array}$ & $\begin{array}{l}-0.014 \\
(0.45)\end{array}$ & $\begin{array}{l}0.428 \\
(0.3)\end{array}$ & $\begin{array}{c}0.002 \\
(0.45)\end{array}$ & $\begin{array}{l}-0.019 \\
(0.45)\end{array}$ & $\begin{array}{l}0.359 \\
(0.3)\end{array}$ & $\begin{array}{l}-0.001 \\
(0.45)\end{array}$ \\
\hline 26 & 0.346 & 0.017 & 0.286 & 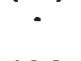 & $\begin{array}{c}0.498 \\
(0.25)\end{array}$ & $\begin{array}{r}0.554 \\
(0.25)\end{array}$ & $\begin{array}{l}-0.048 \\
(0.45)\end{array}$ & $\begin{array}{l}-0.027 \\
(0.45)\end{array}$ & $\begin{array}{r}0.886 \\
(0.25)\end{array}$ & $\begin{array}{l}0.066 \\
(0.4)\end{array}$ & $\begin{array}{l}-0.024 \\
(0.45)\end{array}$ & $\begin{array}{l}-0.045 \\
(0.45)\end{array}$ & $\begin{array}{r}-0.03 \\
(0.45)\end{array}$ & $\begin{array}{l}-0.023 \\
(0.45)\end{array}$ & $\begin{array}{l}-0.005 \\
(0.45)\end{array}$ & $\begin{array}{l}-0.005 \\
(0.45)\end{array}$ & $\begin{array}{l}0.287 \\
(0.3)\end{array}$ & $\begin{array}{l}-0.024 \\
(0.45)\end{array}$ & $\begin{array}{c}0.002 \\
(0.45)\end{array}$ & $\begin{array}{l}-0.007 \\
(0.45)\end{array}$ \\
\hline 27 & 0.467 & -0.014 & 0.298 & 0.222 & - & $\begin{array}{c}3.523^{n} \\
(0.1)\end{array}$ & $\begin{array}{l}-0.049 \\
(0.45)\end{array}$ & $\begin{array}{l}0.579 \\
(0.3)\end{array}$ & $\begin{array}{c}0.04 \\
(0.4)\end{array}$ & $\begin{array}{l}-0.027 \\
(0.45)\end{array}$ & $\begin{array}{l}0.248 \\
(0.3)\end{array}$ & $\begin{array}{l}0.071 \\
(0.3)\end{array}$ & $\begin{array}{l}-0.005 \\
(0.45)\end{array}$ & $\begin{array}{l}-0.013 \\
(0.45)\end{array}$ & $\begin{array}{l}-0.004 \\
(0.45)\end{array}$ & $\begin{array}{l}-0.022 \\
(0.45)\end{array}$ & $\begin{array}{l}-0.001 \\
(0.45)\end{array}$ & $\begin{array}{r}0.038 \\
(0.35)\end{array}$ & $\begin{array}{l}0.193 \\
(0.3)\end{array}$ & $\begin{array}{l}-0.031 \\
(0.45)\end{array}$ \\
\hline 28 & -0.188 & 0.221 & 0.024 & -0.009 & $\begin{array}{c}-0.705 \\
\left(n^{n *}\right)\end{array}$ & . & $\begin{array}{l}-0.019 \\
(0.45)\end{array}$ & $\begin{array}{l}-0.027 \\
(0.45)\end{array}$ & $\begin{array}{l}0.206 \\
(0.3)\end{array}$ & $\begin{array}{l}0.393 \\
(0.25)\end{array}$ & $\begin{array}{r}0.063 \\
(0.35)\end{array}$ & $\begin{array}{l}-0.035 \\
(0.45)\end{array}$ & $\begin{array}{l}-0.026 \\
(0.45)\end{array}$ & $\begin{array}{l}-0.045 \\
(0.45)\end{array}$ & $\begin{array}{l}0.134 \\
(0.3)\end{array}$ & $\begin{array}{l}-0.031 \\
(0.45)\end{array}$ & $\begin{array}{l}-0.011 \\
(0.45)\end{array}$ & $\begin{array}{l}-0.019 \\
(0.45)\end{array}$ & $\begin{array}{c}0.719 \\
(0.25)\end{array}$ & $\begin{array}{l}-0.024 \\
(0.45)\end{array}$ \\
\hline 29 & 0.244 & -0.063 & 0,000 & 0.186 & 0.455 & -0.128 & - & $\begin{array}{r}0.004 \\
(0.45)\end{array}$ & $\begin{array}{l}0.613 \\
(0.3)\end{array}$ & $\begin{array}{c}0.129 \\
(0.35)\end{array}$ & $\begin{array}{l}-0.093 \\
(0.45)\end{array}$ & $\begin{array}{l}-0.019 \\
(0.45)\end{array}$ & $\begin{array}{l}-0.013 \\
(0.45)\end{array}$ & $\begin{array}{l}-0.057 \\
(0.45)\end{array}$ & $\begin{array}{l}-0.014 \\
(0.45)\end{array}$ & $\begin{array}{l}0.415 \\
(0.3)\end{array}$ & $\begin{array}{l}0.048 \\
(0.4)\end{array}$ & $\begin{array}{l}0.066 \\
(0.4)\end{array}$ & $\begin{array}{r}0.396 \\
(0.35)\end{array}$ & $\begin{array}{l}0.161 \\
(0.35)\end{array}$ \\
\hline 30 & -0.214 & -0.129 & 0.208 & 0.208 & 0.248 & -0.348 & 0.042 & - & $\begin{array}{l}-0.048 \\
(0.45)\end{array}$ & $\begin{array}{l}-0.017 \\
(0.45)\end{array}$ & $\begin{array}{r}0.269 \\
(0.35)\end{array}$ & $\begin{array}{l}0.092 \\
(0.4)\end{array}$ & $\begin{array}{r}0.754 \\
(0.25)\end{array}$ & $\begin{array}{l}-0.042 \\
(0.45)\end{array}$ & $\begin{array}{l}-0.006 \\
(0.45)\end{array}$ & $\begin{array}{l}0.089 \\
(0.4)\end{array}$ & $\begin{array}{c}-0.064 \\
(0.45)\end{array}$ & $\begin{array}{l}-0.006 \\
(0.45)\end{array}$ & $\begin{array}{l}-0.084 \\
(0.45)\end{array}$ & $\begin{array}{l}-0.017 \\
(0.45)\end{array}$ \\
\hline 15 & -0.196 & -0.355 & -0.022 & 0.095 & -0.008 & -0.232 & -0.080 & 0.272 & - & $\begin{array}{l}-0.015 \\
(0.45)\end{array}$ & $\begin{array}{l}-0.024 \\
(0.45)\end{array}$ & $\begin{array}{l}-0.068 \\
(0.45)\end{array}$ & $\begin{array}{l}-0.014 \\
(0.45)\end{array}$ & $\begin{array}{l}-0.062 \\
(0.45)\end{array}$ & $\begin{array}{r}0.01 \\
(0.45)\end{array}$ & $\begin{array}{l}-0.031 \\
(0.45)\end{array}$ & $\begin{array}{l}-0.015 \\
(0.45)\end{array}$ & $\begin{array}{l}-0.033 \\
(0.45)\end{array}$ & $\begin{array}{r}0.06 \\
(0.4)\end{array}$ & $\begin{array}{l}0.091 \\
(0.4)\end{array}$ \\
\hline 46 & 0.156 & 0.327 & 0.025 & 0.228 & -0.247 & 0.258 & -0.077 & -0.033 & -0.015 & - & $\begin{array}{l}-0.013 \\
(0.45)\end{array}$ & $\begin{array}{l}-0.002 \\
(0.45)\end{array}$ & $\begin{array}{r}0.004 \\
(0.45)\end{array}$ & $\begin{array}{l}-0.003 \\
(0.45)\end{array}$ & $\begin{array}{l}-0.018 \\
(0.45)\end{array}$ & $\begin{array}{l}-0.007 \\
(0.45)\end{array}$ & $\begin{array}{r}0.238 \\
(0.35)\end{array}$ & $\begin{array}{l}-0.038 \\
(0.45)\end{array}$ & $\begin{array}{r}0.003 \\
(0.45)\end{array}$ & $\begin{array}{l}0.019 \\
(0.4)\end{array}$ \\
\hline 47 & 0.191 & 0.067 & 0.125 & 0.016 & 0.614 & -0.250 & 0.199 & -0.008 & -0.145 & 0.114 & - & $\begin{array}{r}0.226 \\
(0.35)\end{array}$ & $\begin{array}{r}0.007 \\
(0.45)\end{array}$ & $\begin{array}{r}0.005 \\
(0.45)\end{array}$ & $\begin{array}{l}-0.006 \\
(0.45)\end{array}$ & $\begin{array}{r}0.005 \\
(0.45)\end{array}$ & $\begin{array}{l}-0.007 \\
(0.45)\end{array}$ & $\begin{array}{l}-0.022 \\
(0.45)\end{array}$ & $\begin{array}{r}0.361 \\
(0.35)\end{array}$ & $\begin{array}{l}-0.022 \\
(0.45)\end{array}$ \\
\hline 48 & 0.073 & 0.344 & 0.103 & 0.014 & 0.264 & 0.545 & 0.240 & -0.044 & -0.115 & 0.308 & 0.330 & - & $\begin{array}{l}0.149 \\
(0.4)\end{array}$ & $\begin{array}{r}-0.045 \\
(0.45)\end{array}$ & $\begin{array}{l}-0.014 \\
(0.45)\end{array}$ & $\begin{array}{r}0.214 \\
(0.35)\end{array}$ & $\begin{array}{r}0.147 \\
(0.35)\end{array}$ & $\begin{array}{r}-0.015 \\
(0.45)\end{array}$ & $\begin{array}{r}0.004 \\
(0.45)\end{array}$ & $\begin{array}{l}0.036 \\
(0.4)\end{array}$ \\
\hline 49 & -0.155 & 0.062 & -0.217 & -0.101 & 0.142 & 0.541 & 0.062 & -0.041 & -0.282 & 0.149 & 0.336 & 0.202 & - & $\begin{array}{l}-0.007 \\
(0.45)\end{array}$ & $\begin{array}{c}0.08 \\
(0.35)\end{array}$ & $\begin{array}{r}0.545 \\
(0.25)\end{array}$ & $\begin{array}{r}0.005 \\
(0.45)\end{array}$ & $\begin{array}{r}0.072 \\
(0.35)\end{array}$ & $\begin{array}{r}0.171 \\
(0.35)\end{array}$ & $\begin{array}{l}-0.041 \\
(0.45)\end{array}$ \\
\hline 111 & 0.143 & -0.046 & -0.093 & -0.072 & -0.103 & 0.167 & -0.058 & -0.031 & 0.167 & -0.133 & -0.075 & -0.161 & 0.069 & - & $\begin{array}{r}0.061 \\
(0.35)\end{array}$ & $\begin{array}{l}-0.071 \\
(0.45)\end{array}$ & $\begin{array}{l}-0.048 \\
(0.45)\end{array}$ & $\begin{array}{l}-0.007 \\
(0.45)\end{array}$ & $\begin{array}{l}0.031 \\
(0.4)\end{array}$ & $\begin{array}{l}0.382 \\
(0.3)\end{array}$ \\
\hline 112 & -0.188 & 0.827 & -0.054 & -0.203 & $0.754^{\star \prime}$ & -0.194 & 0.259 & 0.168 & -0.185 & -0.159 & 0.069 & 0.485 & 0.487 & -0.086 & . & $\begin{array}{l}0.166 \\
(0.3)\end{array}$ & $\begin{array}{l}-0.028 \\
(0.45)\end{array}$ & $\begin{array}{l}-0.017 \\
(0.45)\end{array}$ & $\begin{array}{l}0.009 \\
(0.4)\end{array}$ & $\begin{array}{l}-0.031 \\
(0.45)\end{array}$ \\
\hline 113 & 0,000 & 0.260 & 0.226 & -0.037 & -0.066 & 0.630 & -0.020 & 0.150 & 0.095 & -0.317 & -0.218 & 0.010 & -0.056 & -0.083 & 0.451 & - & $\begin{array}{r}0.189 \\
(0.35)\end{array}$ & $\begin{array}{l}0.047 \\
(0.35)\end{array}$ & $\begin{array}{l}0.422 \\
(0.3)\end{array}$ & $\begin{array}{l}0.001 \\
(0.45)\end{array}$ \\
\hline 114 & 0.380 & -0.176 & 0.190 & 0.000 & 0,000 & 0,000 & 0.123 & 0,000 & -0.102 & -0.126 & 0.130 & 0.193 & 0.201 & 0.090 & 0,000 & 0,000 & - & $\begin{array}{l}-0.054 \\
(0.45)\end{array}$ & $\begin{array}{l}0.103 \\
(0.4)\end{array}$ & $\begin{array}{r}-0.06 \\
(0.45)\end{array}$ \\
\hline 178 & 0.035 & -0.187 & 0.572 & 0.083 & 0.591* & $-0.632^{n}$ & 0.311 & 0.052 & -0.204 & -0.006 & 0.475 & 0.595 & -0.038 & -0.266 & 0.167 & -0.191 & 0.123 & - & $\begin{array}{l}-0.072 \\
(0.45)\end{array}$ & $\begin{array}{l}-0.027 \\
(0.45)\end{array}$ \\
\hline 179 & 0.023 & 0.150 & -0.040 & -0.026 & -0.018 & 0.114 & -0.015 & -0.001 & -0.051 & -0.009 & 0.081 & -0.068 & 0.193 & -0.003 & -0.050 & 0.087 & 0,000 & -0.084 & - & $\begin{array}{l}0.022 \\
(0.4)\end{array}$ \\
\hline 180 & -0.097 & -0.111 & 0.077 & 0.185 & 0.158 & -0.036 & 0.164 & 0.064 & 0.017 & 0.091 & 0.098 & 0.050 & 0.071 & -0.004 & -0.056 & -0.100 & 0.111 & 0.172 & 0.006 & - \\
\hline
\end{tabular}

Above the diagonal: maximum LOD scores between pairs of loci estimated by summing single estimates over 'informative' families of megagametophytes, i.e. from doubly heterozygous mother trees (in parentheses, recombination fraction $\theta_{i}$ at which the maximum LOD scores were estimated). Under the diagonal: linkage disequilibrium coefficients $D^{\prime}\left(D / D_{\max }\right)$ calculated from genotype frequencies. Disequilibrium was significantly different from zero only for 5 out of 190 pairs of RAPD loci.

${ }^{*} P<0.05, * * P<0.01,{ }^{* * *} P<0.001$.

values of s.n.d. $(\alpha<0.05)$ out of 355 total genotypic pairs over the five distance classes identified. Four of these values were discarded a priori because of an expected number of joins lower than 1 . Out of the other 30 cases, 12 involved homozygous pairs ('11_11, '11_00', '00_00'), 13 homo-heterozygous pairs ('11_10', '10_00'), and five heterozygous pairs ('10_10'). Overall, a slightly decreasing trend in the proportion of positive, significant s.n.d. values in the first three distance classes was detected for homotypic pairs $(0.13,0.10$, 0.03 , respectively) as well as a decrease in negative s.n.d. values for heterotypic pairs $(0.06,0.03,0.00$, respectively). For both these kinds of interactions a peak in the proportion of significant s.n.d. values was observed in the fourth distance class $(0.13$ and 0.09 , respectively), probably for stochastic reasons.

A nonrandom spatial distribution was observed for some single-locus genotype pairs. Figure 3 shows the spatial patterns detected, grouped by genotypic pair type. Markers 27 and 49 showed an excess of homozygotes for short distances, while marker 29 showed a deficiency of the heterotypic pair ' $11 \_00$ ' for the first distance class (Fig. 3a). On the other hand, RAPD markers 23,24, 28 and 46 showed an excess of heterozygotes on the short distances (Figs $3 \mathrm{~b}$ and $3 \mathrm{c}$ ).

$\mathrm{Di}$ - and trilocus spatial autocorrelation analysis was carried out only on loci showing linkage disequilibrium (see above). Significant values of s.n.d. were observed (Fig. 3d) for combinations 27/28 (dilocus genotypic pair: ' $10 / 10 \_10 / 10$ ') and 27/178 (dilocus genotypic pair: '10/00 10/00') for the shortest distance classes, but none of the relative correlograms was significant by the Bonferroni test $\left(P_{\text {[adjusted }}>0.05\right)$. Moreover, an interesting although nonsignificant trend was found for combinations 28/178 (dilocus genotypic pair: "10/ $00 \_10 / 00^{\prime}$ ) and 27/28/178 (trilocus genotypic pair: '10/ 

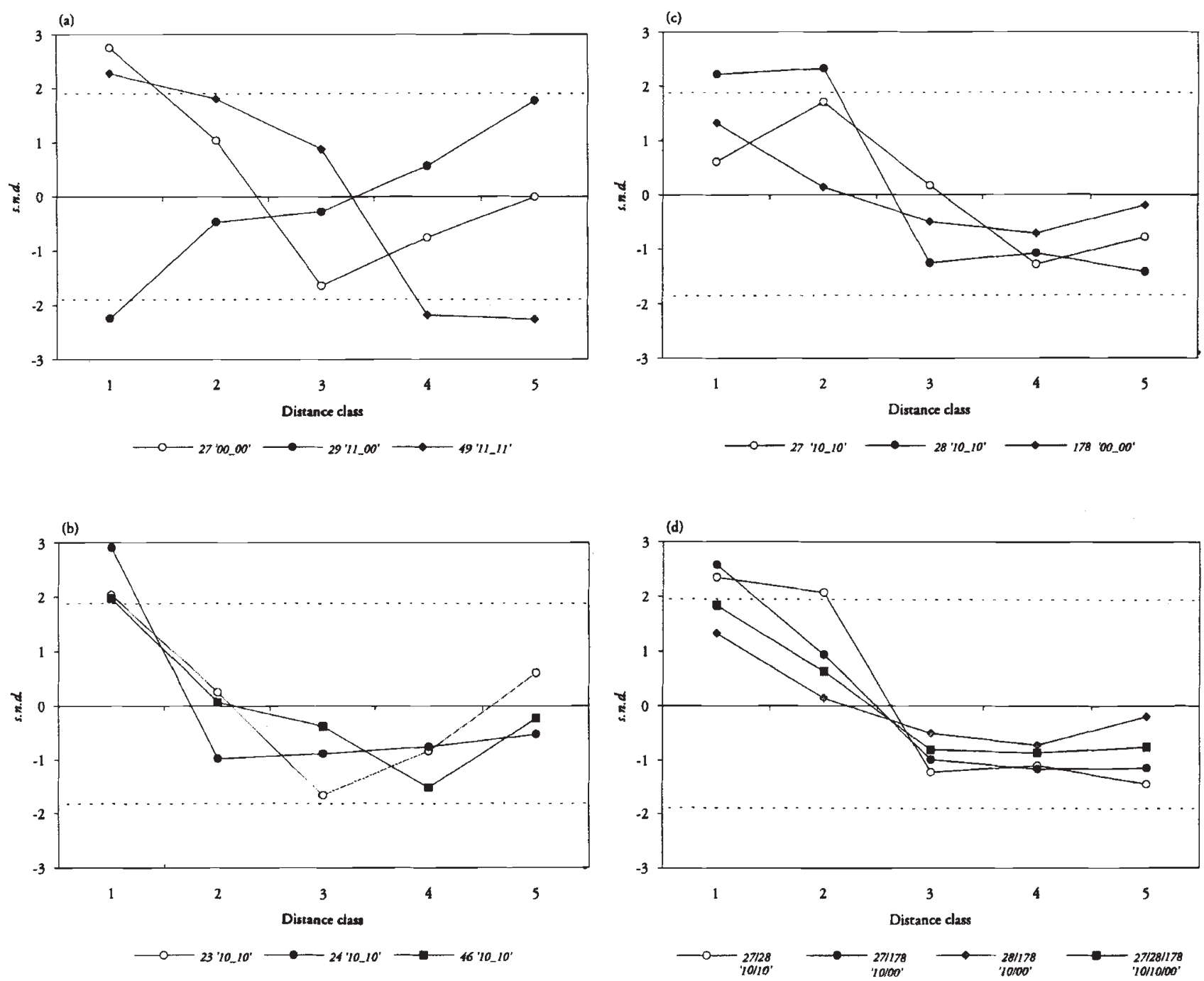

Fig. 3 Spatial autocorrelation analysis of the Campolino Norway spruce population. (a) Single-locus correlograms for the homozygotic pairs: marker $27^{\prime} 00 \_00^{\prime}$, marker $29^{\prime} 11 \_00^{\prime}$ and marker 49'11_11'. (b) Single-locus correlograms for the heterozygotic pairs: marker $23^{\prime} 10 \_10^{\prime}$ ', marker $24^{\prime} 10_{-} 10^{\prime}$ ' and marker $46^{\prime} 10_{-} 10^{\prime}$. (c) Single-locus correlograms for the homotypic pairs $27^{\prime} 10 \_10^{\prime}, 28^{\prime} 10 \_10^{\prime}$ ' and $178^{\circ} 00 \_00^{\prime}$. (d) Di- and trilocus correlograms for the homotypic pairs $27 / 28^{\circ} 10 / 10 \_10 / 10^{\prime}$ and $27 / 178$ '10/00_10/00', 28/178'10/00_10/00' and 27/28/178'10/10/00_10/10/00'.

$\left.10 / 00-10 / 10 / 00^{\prime}\right)$. None of the other di- or trilocus allelic combinations at these or other loci showing significant disequilibrium (23/24 and 27/112) showed significant correlograms.

\section{Discussion}

\section{Segregation analysis and linkage relationships over the whole population}

Segregation analysis over the whole population confirms previously reported evidence on the genetic origin of the RAPD marker bands in Picea abies (Bucci \& Menozzi, 1993; Bucci et al., 1995). The good fit of the observed segregation ratios within families to the expected binomial distribution is consistent with a simple, dominant, Mendelian fashion of inheritance of RAPD markers. Our data are a further contribution to the study of the reliability and stability of RAPD markers in different genomic backgrounds (Heun \& Helentjaris, 1993; Isabel et al., 1993; Levitan \& Grosberg, 1993).

In this study we analysed recombination fractions from family (megagametophyte) data and estimated 
disequilibrium from genotype data. Linkage analysis of family data confirmed the linkage relationships between RAPD markers previously reported (Binelli \& Bucci, 1994). The recombination fraction estimated over the population between markers $27 / 28$ was significantly larger ( $\mathrm{rf}=0.10$ vs. 0.016 ) than that previously reported, as expected given the low power of the phase-unknown double backcross analysis. It has also been reported that the loss of information is relatively larger at larger values of recombination fraction (Ott, 1991): this may explain the failure to detect linkage between the pair of loci 24/178.

Very few cases of linkage disequilibrium between loci in forest tree species have been described in the literature. Epperson \& Allard (1987) analysed 14 allozymic loci in Pinus contorta, detecting a large disequilibrium between some tightly linked loci $(P E R-1 / P E R-2$, rf $<0.002 ; \quad G O T-1 / P E R-1$ and GOT-1/PER-2, $\mathrm{rf}=0.007)$ and no disequilibrium for other linked loci (PGI-2/PER-1, rf =0.013; PGI-2/PER-2, $\mathrm{rf}=0.013$; $G O T-1 / P G I-2, \mathrm{rf}=0.020)$. The authors stated also that linkage alone did not completely account for the large disequilibrium detected. Mitton and collegues (1980) analysed seven allozymic loci in three populations along an elevational gradient, detecting an excess for five (16.7 per cent) out of 30 possible genotypes for pairs of loci for which the linkage relationship was unknown. Almost all of the significant values involved loci $P E R$ or $P G M$, for which they found some evidence of natural selection. Finally, Yang \& Yeh (1993) found an extensive gametic disequilibrium in 40 out of 60 populations of three subspecies of Pinus contorta, thought to result from a multilocus Wahlund effect after fire establishment.

In this study, the proportion of significant $D$ values estimated from genotypic frequencies ( 2.63 per cent) is lower than what is expected by chance alone. The large disequilibrium detected over the population between loci $27 / 28$ seems to result from tight linkage, as expected from theoretical considerations (Hill \& Robertson, 1968; Hedrick, 1994). On the other hand, previously detected linked markers, like $25 / 26$ or $24 /$ 178 , showed very low, nonsignificant $D$ values, suggesting a large recombination rate for pairs of loosely linked loci - as expected for allogamous species characterized by large gene flow like Norway spruce - and no epistatic selection.

\section{Estimation of the population parameters and spatial genetic structure}

In this study, most of the markers analysed showed an excess of heterozygotes. Mean observed (0.381) and expected (0.338) heterozygosity was higher than estimates obtained from allozymes for the Campolino
Norway spruce population. Giannini and colleagues (1991) reported an estimate of mean expected heterozygosity for the Campolino stand of $0.199 \pm 0.051$ analysing 17 allozymic loci (four monomorphic), with large values at some of the loci studied (GOT$B=0.557 ; L A P-B=0.522 ; N D H-A=0.455 ; 6 P G D-$ $C=0.447$; etc.). Boscherini and colleagues (1993), studying four allozymic loci in the same population, found an average value for Wright's inbreeding coefficient $\left(F_{\text {IS }}\right)$ of $-0.110 \pm 0.094$ for the adult stage, slightly lower than the estimate obtained in this study (-0.136). Lagercrantz \& Ryman (1990), in the largest investigation carried out on Picea abies (70 populations, 22 loci), found an average $H_{\text {obs }}=0.115$. On the other hand, Tigerstedt (1973) referred to an average observed heterozygosity of 0.430 detected by monitoring four allozymic loci ( 2.5 alleles per locus) in two natural populations. Lundkvist (1979) reported an average $H_{\text {obs }}=0.360$ obtained by screening 11 allozymic loci in four populations of Norway spruce.

Most of the relatively few papers on spatial autocorrelation in natural populations of forest trees report only a weak spatial genetic structure (e.g., Epperson \& Allard, 1989; Knowles, 1991; Perry \& Knowles, 1991; Merzeau et al., 1994, etc.). On the other hand, Knowles \& Perry (1992), monitoring only three allozymic loci, observed remarkable differences in the genetic structure between two 20-year-old populations of Larix laricina with different establishment histories (growing in a clear-cut area within the forest and in a colonized abandoned hay field). Wagner et al. (1991) reported a strong autocorrelation between some chloroplastic variants through the hybrid zone of Pinus contorta and Pinus banksiana.

In this study a weak spatial structure at the scale considered $(0-180 \mathrm{~m})$ was detected. The number of positive, significant s.n.d. values for homotypic pairs over all loci for short distances was only slightly greater than expected by chance ( 5 per cent) and genetic similarity did not significantly decrease with distance. This seems to suggest a lack of 'family structure' arising from isolation by distance (a cluster of individuals correlated over most loci - Wright, 1943), as expected for a species characterized by large outcrossing rates $(t \sim 0.90$; Müller, 1977).

On the other hand, we found an excess over short distances of single-locus homozygotes (markers 27 and 49 ) as well as single-locus heterozygotes (markers 23, 24 and 46). This suggests the existence within the population of clusters of individuals with some degree of correlation (the mean dimension of these patches was 40-50 metres, except for marker 49 80 meters).

Multilocus spatial autocorrelation carried out on loci 27,28 and 178 detected an interesting (although 
nonsignificant) aggregation of similar multilocus genotypes. Lowered recombination over the whole population between these markers, because of tight linkage and/or strong gametic disequilibrium (see above), could reasonably account for the dilocus structure detected. On the other hand, linkage cannot account for the dilocus structure involving markers 27 and 178 , as well as for the gametic disequilibrium between them. Further analyses are needed to throw light on the causes of the described genetic structure.

Small-scale spatial genetic structure within populations of forest trees has been claimed to affect the level of inbreeding and the action of natural selection (Epperson, 1990). Spatial aggregation of particular allelic configurations can be considered the first step towards the identification of interacting sets of loci involved in the microevolutionary processes (Allard, 1975). Furthermore, analysis of the covariation along major environmental gradients of the frequencies of multilocus genotypes and environmental parameters could be a useful approach to the study of selective forces involved in microevolutionary processes.

\section{Acknowledgements}

We thank Dr S. Leonardi (University of Parma, Italy) for the use of the spatial autocorrelation programs and for helpful discussion of the results. We are also grateful to Professor R. Giannini (University of Florence, Italy) for sharing with us his knowledge of the Campolino stand. This research was supported by the National Research Council of Italy (Comitato Ambiente, C.N.R.).

\section{References}

ALLARD, R. W. 1975. The mating system and microevolution. Genetics, 79, 115-126.

ALLARD, R. W., BABBEL, G. R., CLEGG, M. T. AND KAHLER, A. L. 1972. Evidence of coadaptation in Avena barbata. Proc. Natl. Acad.Sci., U.S.A., 69, 3043-3048.

AYLIFFE, M. A., LAWRENCE, G. J., ELLIS, J. G. AND PRYOR, A. J. 1994. Heteroduplex molecules formed between allelic sequences cause nonparental RAPD bands. $\mathrm{Nucl}$. Acids Res., 22, 1632-1636.

BERGMANN, F. 1991. Isozyme gene markers. In: Müller-Starck, G. and Ziehe, M., (eds) Genetic Variation in European Populations of Forest Trees, pp. 67-78. Sauerlander Verlag, Frankfurt am Main.

BINELLI, G. AND BUCCI, G. 1994. A genetic linkage map of Picea abies Karst. based on RAPD markers as a tool in population genetics. Theor. Appl. Genet., 88, 283-288.

BOSCHERINI, G., VENDRAMIN, G. G. AND GIANNINI, R. 1993. Mating system analysis of two Italian populations of Norway spruce. J. Genet. Breed., 47, 45-48.
BUCCI, G., BINELLI, G. AND MENOZZI, P. 1995. Identification of a new set of molecular markers in Picea abies Karst., as revealed by random amplification techniques. In: Baradat, Ph. (ed.) Population Genetics and Gene Conservation. SPB Academic Publishing bv, The Hague, The Netherlands (in press).

BUCC, G. AND MENOZZI, P. 1993. Segregation analysis of random amplified polymorphic DNA (RAPD) markers in Picea abies Karst. Mol. Ecol., 2, 227-232.

CESARONE, C. F., BOLOGNESI, C. AND SANTI, L. 1979. Improved microfluorometric DNA determination in biological material using 33258 Hoechst. Analyt. Biochem., 100, 188-197.

CLIFF, A. D. AND ORD, J. K. 1973. Spatial Autocorrelation. Pion, London.

COCKERHAM, C. C. AND WEIR, B. S. 1977. Digenic descent measures in a finite population. Genet. Res., 30, 121-147.

ELLSWORTH, D. L., RITTENHOUSE, K. D. AND HONEYCUTT, R. L. 1993. Artifactual variation in randomly amplified polymorphic DNA banding patterns. BioTechniques, 14, 214-217.

EPPERSON, B. K. 1990. Spatial patterns of genetic variation within plant populations. In: Brown, A. H. D., Clegg, M. T., Kahler, A. L. and Weir, B. S. (eds) Plant Population Genetics, Breeding, and Genetic Resources, pp. 254-278. Sinauer, Sunderland, MA.

EPPERSON, B. K. AND ALLARD, R. w. 1987. Linkage disequilibrium between allozymes in natural populations of lodgepole pine. Genetics, 115, 341-352.

EPPERSON, B. K. AND ALLARD, R. W. 1989. Spatial autocorrelation analysis of the distribution of genotypes within populations of lodgepole pine. Genetics, 121, 369-377.

GERBER, S. AND RODOLPHE, F. 1994. Estimation and test for linkage between markers: a comparison of lod score and $\chi^{2}$ test in a linkage study of maritime pine (Pinus pinaster Ait. ). Theor. Appl. Genet., 88, 293-297.

GIANNINI, R., MORGANTE, M. AND VENDRAMIN, G. G. 1991. Allozyme variations in Italian populations of Picea abies. Silvae Genet., 40, 160-166.

HADRYS, H., BALICK, M. AND SCHIERWATER, B. 1992. Applications of random amplified polymorphic DNA (RAPD) in molecular ecology. Mol. Ecol., 1, 55-63.

HAMRICK, J. L. AND ALLARD, R. W. 1972. Microgeographical variation in allozyme gene frequencies in Avena barbata. Proc. Natl. Acad. Sci. U.S.A., 69, 2100-2104.

HASTINGS, A. 1990. The interaction between selection and linkage in plant populations. In: Brown, A. H. D., Clegg, M. T., Kahler, A. L. and Weir, B. S. (eds) Plant Population Genetics, Breeding and Genetic Resources, pp. 163-180. Sinauer, Sunderland, MA.

HEDRICK, P. W. 1994. Evolutionary genetics of the major histocompatibility complex. Am. Nat., 143, 945-964.

HEUN, M. AND HELENTJARIS, T. 1993. Inheritance of RAPDs in $\mathrm{F}_{1}$ hybrids of corn. Theor. Appl. Genet., 85, 961-968.

HILL, W. G. AND ROBERTSON, A. 1968. Linkage disequilibrium in finite populations. Theor. Appl. Genet., 38, 226-231.

ISABEL, N., TREMBLAY, L., MICHAUD, M., TREMBLAY, F. AND BOUSQUET, J. 1993. RAPDs as an aid to evaluate the genetic integrity of somatic embryogenesis-derived populations of Picea mariana (Mill.) B. S. P. Theor. Appl. Genet., 86, 81-87.

(c) The Genetical Society of Great Britain, Heredity, 75, 188-197. 
KNOWLES, P. 1991. Spatial genetic structure within two natural stands of black spruce (Picea mariana (Mill.) B.S.P.). Silvae Genet., 40, 13-19.

KNOWLES, P. AND PERRY, D. J. 1992. Spatial genetic structure in two tamarack (Larix laricina (Du Roi) K. Koch) populations with differing establishment histories. Evolution, 46, 572-576.

KREITMANN, M. 1983. Nucleotide polymorphism at the alcohol dehydrogenase locus of Drosophila melanogaster. Nature, 304, 412-417.

LAGERCRANTZ, U. AND RYMAN, N. 1990. Genetic structure of Norway spruce (Picea abies): concordance of morphological and allozymic variation. Evolution, 44, 38-53.

LEIGH BROWN, A. J. 1989. Population genetics at the DNA level: a review of contribution of restriction enzyme studies. Oxford Surv. Evol. Biol., 6, 207-242.

LEVITAN, D. R. AND GRoSBERG, R. K. 1993. The analysis of paternity and maternity in the marine hydrozoan Hydractinia symbiolongicarpus using random amplified polymorphic DNA (RAPD) markers. Mol. Ecol., 2, 315-326.

LUNDKVIST, K. 1979. Allozyme frequency distributions in four Swedish populations of Norway Spruce (Picea abies K.) I. Estimations of genetic variation within and among populations, genetic linkage and a mating system parameter. Hereditas, 90, 127-143.

MATHER, K. 1951. The Measurement of Linkage in Heredity. Methuen, London.

MERZEAU, D., COMPS, B., THIÉBAUT, B., CUGUEN, J. AND LETOUZEY, J. 1994. Genetic structure of natural stands of Fagus sylvatica L. (beech). Heredity, 72, 269-277.

MITTON, J. B., STURGEON, K. B. AND DAVIS, M. c. 1980. Genetic differences in ponderosa pine along a steep elevational gradient. Silvae Genet., 29, 100-103.

MORTON, N. E. 1955. Sequential tests for the detection of linkage. Am. J. Hum. Genet., 7, 277-318.

MÜLLER, G. 1977. Cross-fertilization in a conifer stand inferred from enzyme gene-markers in seeds. Silvae Genet., 26, 223-226.

MÜLlER-STARCK, G., BARADAT, PH. AND BERGMANN, F. 1992. Genetic variation within European tree species. New Forests, 6, 23-47.

NEALE, D. B. AND SEDEROFF, R. R. 1991. Genome mapping in pines takes shape. Probe, 1, 1-3.

NEI, M. 1987. Molecular Evolutionary Genetics. Sinauer, Sunderland, MA.

NELSON, C. D., NANCE, W. L. AND DOUDRICK, R. L. 1993. A partial genetic linkage map of slash pine (Pinus elliottii Engelm. var. elliottii) based on random amplified polymorphic DNAs. Theor. Appl. Genet., 87, 145-151.
OTT, J. 1991. Analysis of Human Genetic Linkage, Revised edn. Johns Hopkins University Press, Baltimore.

PARAN, I., KESSELI, R. V. AND MICHELMORE, R. W. 1991. Identification of restriction fragment length polymorphisms and random amplified polymorphic DNA markers linked to downy mildew resistance genes in lettuce, using nearisogenic lines. Genome, 34, 1021-1027.

PERRY, D. J. AND KNOWLES, P. 1991. Spatial genetic structure within three sugar maple (Acer saccharum Marsh.) stands. Heredity, 66, 137-142.

REITER, R. S., WILLIAMS, J. G. K., FELDMANN, K. A., RAFALSKI, J. A., TINGEY, S. V. AND SCOLNIK, P. A. 1992. Global and local genome mapping in Arabidopsis thaliana by using recombinant inbred lines and random amplified polymorphic DNAs. Proc. Natl. Acad. Sci. U.S.A., 89, 1477-1481.

SLATKIN, M. AND ARTER, H. E. 1991. Spatial autocorrelation methods in population genetics. Am. Nat., 138, 499-517.

SOKAL, R. R. AND ODEN, N. L. 1978. Spatial autocorrelation in biology. 1. Methodology. Biol. J. Linn. Soc., 10, 199-228.

TIGERSTEDT, P. M. A. 1973. Studies of isozyme variation in marginal and central populations of Picea abies. Hereditas, 75, 47-60.

TUlSIERAM, L. K., GLAUBiTZ, J. C., KISS, G. AND CARLSON, J. E. 1992. Single-tree genetic linkage mapping in conifers using haploid DNA from megagametophytes. Bio/Technology, 10, 686-690.

WAGNER, D. B. 1992. Nuclear, chloroplast, and mitochondrial DNA polymorphisms as biochemical markers in population analyses of forest trees. New Forests, 6, 373-390.

WAGNER, D. B., FURNIER, G. R., SAGHAI-MAROOF, M. A., WILLIAMS, S. M., DANCIK, B. P. AND ALLARD, R. W. 1987. Chloroplast DNA polymorphisms in lodgepole and jack pines and their hybrids. Proc. Natl. Acad. Sci. U.S.A., 84, 2097-2100.

WAGNER, D. B., SUN, Z.-X., GOVINDARAJU, D. R. AND DANCIK, B. P. 1991. Spatial patterns of chloroplast DNA and cone morphology variation within populations of a Pinus banksiana-Pinus contorta sympatric region. Am. Nat., 138, 156-170.

WeIR, B. S. 1990. Genetic Data Analysis. Sinauer, Sunderland, MA.

WILLIAMS, J. G. K., KUBELIK, A. R., LIVAL, K. J., RAFALSKI, J. A. AND TINGEY, S. V. 1990. DNA polymorphisms amplified by arbitrary primers are useful as genetic markers. Nucl. Acids Res., 18, 6531-6535.

WRIGHT, s. 1943. Isolation by distance. Genetics, 28, 114-138.

YANG, R.-C. AND YEH, F. C. 1993. Multilocus structure in Pinus contorta Dougl. Theor. Appl. Genet., 87, 568-576. 